\title{
Comparison of Brain-derived Neurotrophic Factor Level in Depressed Patients Treated with Fluoxetine and Sertraline
}

\author{
Sonny Teddy Lisal, Nur Aeni M. A. Fattah, Rahmawati Nur Indah*, Saidah Syamsuddin \\ Department of Psychiatry, Faculty of Medicine, Hasanuddin University, Makassar, Indonesia
}

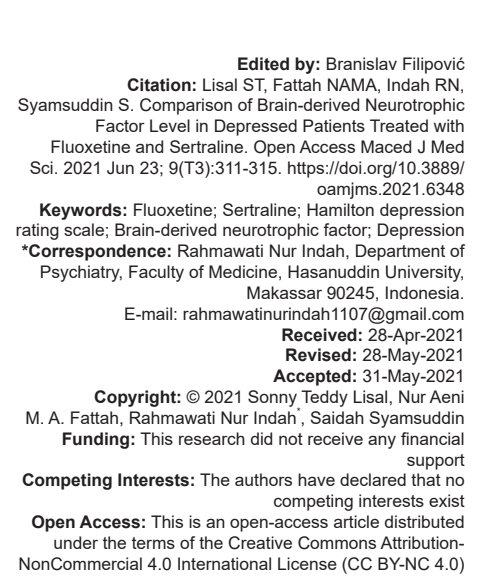

Introduction

Depression is a major contributor of the overall global burden of disease that affects over 300 million people worldwide. Clinical manifestations of depression include one or more persistent episodes of sadness and anhedonia within 2 weeks, with changes in appetite, disturbed sleep patterns, decreased energy levels, decreased physical concentration and activity, feelings of worthlessness, guilt, and thoughts or suicidal behavior. Other symptoms are mostly secondary to changes or have a relationship with these changes. Most of these disorders tend to recur and the occurrence of individual episodes is often associated with significant life events or stressors [1], [2].

Depression is a serious public health concern. The World Health Organization states that depression is in the fourth rank of diseases in the world. Depression affects about $20 \%$ of women and $12 \%$ of men in a lifetime [2], [3]. Based on the results of basic health research in Indonesia (Riskesdas 2013), the prevalence of mental disorders showed by symptoms of depression and anxiety is $6 \%$ for those aged $\geq 15$ years, or about 14 million people [3], [4].
One of the causes of depression is a decrease in neurotrophic activity, one of which is a brain-derived neurotrophic factor (BDNF). Neurotrophic are found in almost all areas of the brain, with the highest levels in the hippocampus and cerebral cortex. These factors play a role in sensory function, perception, motor activity, endocrine regulation, cognition, motivation, and emotion. Neurotropic plays a role in the growth of nerve cells and the differentiation and growth of various forms of neurons in the brain are in the growing stage. In the developed brain (adults), it functions to maintain neurons from damage. Repeated stressors can cause a decrease in BDNF expression. This decrease can cause the death of the pyramid cell layer in the hippocampal CA3 region. In depressed patients, there is a decrease in neurotrophic [5], [6]. The administration of antidepressant drugs can increase BDNF levels, one of which is the SSRI group, by increasing the activation of 5 -HT receptors so that presynaptic serotonin levels increase and activate serotonin receptors [6], [7]. SSRI antidepressants in this case fluoxetine and sertraline have a different mechanism of action in influencing BDNF. The ability of fluoxetine to inhibit serotonin uptake is 23 times stronger than its ability to inhibit 
norepinephrine uptake [6], [7], [8]. One study conducted to see the effect of SSRI on BDNF level showed that sertraline increased the BDNF level after 5 weeks of administration fluoxetine after 6 weeks of administration. Because of these differences, researchers were interested to see which SSRI drugs are the most powerful ones affecting BDNF levels in depressed patients [8], [9], [10].

Fluoxetine is metabolized to norfluoxetine, whose activity is the same as fluoxetine in taking 5-HT. Elimination of half-life of norfluoxetine is longer, which is 4-16 days, while fluoxetine is only 4-6 days. Fluoxetine works to inhibit the reuptake of serotonin neurotransmitters. The structure and activities of each SSRI are different. The chain R-enantiomer of fluoxetine antagonizes the 5-HT 1c receptor at almost micromolar concentrations. Its clinical relevance is unknown. The therapeutic dose of fluoxetine is $20 \mathrm{mg}-80 \mathrm{mg} / \mathrm{day}$ [10], [11].

Serotonin neurons in the midbrain raphe nucleus have autoreceptors on the soma (5-HT1A) and a terminal region (5-HT1B) stimulated by an acute increase in $5-\mathrm{Ht}$ neurons. Sertraline decreases the activity of the sympathetic nervous system. Decreased sympathetic response provides an anxiolytic effect that is associated with the stimulation of 5-HT1A receptors. Sertraline reaches a peak plasma level between 6 and $8 \mathrm{~h}$ after administration. Range of therapeutic doses is 50-200 mg/day [12], [13], [14].

BDNF is the main neuron growth factor in the brain that regulates neurogenesis, neuronal and survival, synaptic maturity, and plasticity. Low BDNF levels are found in the brains of individuals who experience depression, especially in areas (hippocampus, prefrontal cortex, and amygdala) that showed atrophy in depressed patients. Decreased BDNF levels are also found in the blood of patients who are depressed, and this can be reversed by treatment. Negative environmental effects such as psychological stress can also reduce BDNF levels in the hippocampus. The direct impact of antidepressants on BDNF had also been reported, infusion of BDNF into the hippocampus has an antidepressant-like effect. These findings provide hope that increased BDNF levels in certain brain areas targeting the pathways involved can be a new strategy in the prevention and treatment of depression. Research on genetic expression in humans had shown that BDNF is highest in the cortex, hindbrain, and midbrain [14], [15], [16]. Serum BDNF levels are normal in response to some antidepressant therapies [17], [18]. Central reduction in BDNF in specific brain regions has also been reported. A postmortem study in depressed patients reports a reduction in BDNF protein in the hippocampus, along with a reduction in hippocampal volume [18], [19], [20], [21].

\section{Methods}

\section{Patient sample}

The study group subjects were recruited from the outpatient clinic at Wahidin Sudirohusodo Hospital and its affiliates, Makassar, Indonesia. The inclusion criteria are: Male or female subjects with a diagnosis of moderate depression based on the Diagnostic and Statistical Manual of Mental Disorders, Fifth Edition (DSM-V) was treated with fluoxetine or sertraline, either antidepressant naïve, or dropping out of antidepressant therapy for at least 3 months since the last administration, and aged between 18 and 45 years. While the exclusion criteria are: Patients who received any psychotropic medication or suffered from other somatic diseases. Control group were consisted of 10 subjects who were age and sex-matched with the study group, selected among the people visiting psychiatric outpatient department for routine checkup, and Hamilton Depression Rating Scale (HDRS) scored below 7 . The study was conducted from January to February 2019.

\section{Design and procedures}

This study was used an experimental design with a prospective cohort approach that was analyzed before and after 6 weeks of fluoxetine and sertraline administration. Subjects were recruited by consecutive sampling with research requirements that met the inclusion criteria and were willing to take part in this study. All data were recorded, including name, address, gender, age, last education, and history of physical illness. Ethical permission was approved by the committee for Biomedical Research in humans from the Faculty of Medicine at Hasanuddin University and written approval was obtained from all subjects. Three milliliters of peripheral blood samples were taken from each subject to measure BDNF levels before and after 6 weeks of treatment. Blood collection and measurement of BDNF levels and HDRS scores (14-18, Moderate) were measured from each subject before and after 6 weeks of treatment. Measurement of serum BDNF levels was carried out at the Microbiology Laboratory, Faculty of Medicine at Hasanuddin University, using a human brain-derived neurotropic factor kit (R\&D USA) derived from the human brain by the ELISA method.

\section{Statistical analysis}

Data analysis was computed using SPSS version 22. Statistical analysis was performed using Mann-Whitney and Wilcoxon signed-rank and results were statistically significant with $p<0.05$. 


\section{Results}

Based on the statistical analysis, the data included the characteristics and distribution of the subject. The study group involved 20 depressed patients who met the inclusion criteria. The subjects comprised 10 subjects who treated with fluoxetine $20 \mathrm{mg}$ every $24 \mathrm{~h}$ orally and 10 subjects treated with sertraline 50 mg every $24 \mathrm{~h}$ orally. HDRS scores and BDNF levels were measured at the beginning and after 6 weeks of the treatment.

The age of the subjects in the study group was between 20 and 45 years, with mean from fluoxetine group was 35.20 years with standard deviation of 5.31 years and sertraline group was 36.70 years with standard deviation of 7.96 years, whereas the age in the control group was between 19 and 27 years with mean 22.90 years and standard deviation of 2.69 years (Table 1).

Table 1: Descriptive statistic with age group

\begin{tabular}{llllll}
\hline Group & $\mathrm{n}$ & Minimum & Maximum & Mean & Std. Deviation \\
\hline Fluoxetine & 10 & 27 & 43 & 35.20 & 5.31 \\
Sertraline & 10 & 23 & 45 & 36.70 & 7.96 \\
Control & 10 & 19 & 27 & 22.90 & 2.69 \\
\hline
\end{tabular}

Comparison of initial HDRS and BDNF scores between depressed groups and controls was: The HDRS score was significantly higher in the depression group (16.75) than in the control group (3.10) $(p<0.001)$ and BDNF level was significantly lower in the depression group (4.63) than in the control group (13.17) $(p<0.001)$ (Table 2).

Table 2: Comparison of HDRS and BDNF between depression and control at baseline and 6 weeks

\begin{tabular}{llllll}
\hline Variable & Group & $\mathrm{N}$ & Mean & $\mathrm{SD}$ & $\mathrm{p}$ \\
\hline HDRS 0 & Depression & 20 & 16.75 & 1.21 & $<0.001$ \\
& Control & 10 & 3.10 & 1.66 & \\
BDNF 0 & Depression & 20 & 4.63 & 1.11 & $<0.001$ \\
& Control & 10 & 13.17 & 3.82 & \\
\hline BDNF: Brain-derived neurotrophic factor HDRS: Hamilton & depression rating scale.
\end{tabular}

Comparison of HDRS and BDNF level before administration of fluoxetine and sertraline groups is as follows: The HDRS score did not differ significantly between the two groups ( $p>0.05$ ), and BDNF level did not differ significantly between the two groups ( $p>0.05$ ). Comparison of HDRS scores and BDNF level after 6 weeks of fluoxetine and sertraline administration groups are as follows: The HDRS score did not differ significantly between the two groups ( $p>0.05$ ), and BDNF level was significantly higher in the fluoxetine group (9.43) than in the sertraline group $(8.00)(p<0.05)$ (Table 3$)$.

Table 3: Comparison of HDRS score and BDNF level before administration of fluoxetine and sertraline and after 6 weeks administration of fluoxetine and sertraline

\begin{tabular}{llllll}
\hline Variable & Group & $\mathrm{n}$ & Mean & $\mathrm{SD}$ & $\mathrm{p}$ \\
\hline HDRS 0 & Fluoxetine & 10 & 17.20 & 0.92 & 0.116 \\
& Sertraline & 10 & 16.30 & 1.34 & \\
BDNF 0 & Fluoxetine & 10 & 4.70 & 1.19 & 0.762 \\
& Sertraline & 10 & 4.56 & 1.09 & \\
HDRS 6 & Fluoxetine & 10 & 10.40 & 2.59 & 0.647 \\
& Sertraline & 10 & 11.40 & 3.37 & \multirow{2}{*}{0.031} \\
BDNF 6 & Fluoxetine & 10 & 9.43 & 1.56 & \\
& Sertraline & 10 & 8.00 & 1.17 & \\
\hline BDNF: Brain-derived neurotrophic factor, HDRS: Hamilton depression rating scale.
\end{tabular}

Changes in HDRS scores after 6 weeks of therapy compared to baseline were as follows: In the fluoxetine group, there was a significant decrease from 17.20 to 10.40 or a decrease of $39.5 \%$ ( $p<0.01)$; and in the sertraline group, there was a significant decrease from 16.30 to 11.40 or a decrease of $30.1 \%(p<0.01)$. Changes in BDNF expression after 6 weeks of therapy compared to baseline were as follows: In the fluoxetine group, there was a significant increase from 4.70 to 9.43 or an increase of $100.6 \%(p<0.01)$; and in the sertraline group, there was a significant increase from 4.56 to 8.00 or an increase of $75.4 \%(p<0.01)$ (Table 4$)$.

Table 4: Comparison of HDRS score and BDNF level before and after 6 weeks of treatment of fluoxetine and sertraline

\begin{tabular}{llllll}
\hline Group & Variable & $\mathrm{n}$ & Mean & SD & $\mathrm{p}$ \\
\hline Fluoxetine & HDRS 0 & 10 & 17.20 & 0.92 & 0.005 \\
\multirow{2}{*}{ Sertraline } & HDRS 6 & 10 & 10.40 & 2.59 & \\
\multirow{2}{*}{ Fluoxetine } & HDRS 0 & 10 & 16.30 & 1.34 & 0.006 \\
& HDRS 6 & 10 & 11.40 & 3.37 & \\
\multirow{2}{*}{ Sertraline } & BDNF 0 & 10 & 4.70 & 1.19 & 0.005 \\
& BDNF 6 & 10 & 9.43 & 1.56 & \\
& BDNF 0 & 10 & 4.56 & 1.09 & 0.005 \\
\hline HDRS: Hamilton depression rating scale. & 10 & 8.00 & 1.17 & \\
& BDNF 6 & & & &
\end{tabular}

\section{Discussion}

Most literature stated that depression often occurred at a young age, with an average age between 20 and 40 years [1], [2], [3]. Gender of the subjects was mostly women at $10-25 \%$ in both groups, but the distribution of men and women in both groups was not different. Various studies showed that women were twice compared to men with lifetime prevalence in women was $10-25 \%$ and in men was $5-12 \%$. This was under the literature stating that women were more often exposed to environmental stressors and the threshold for stressors was lower in women than men and also related to hormones in women at the time of premenstrual, postpartum, and postmenopause [2], [3], [4]. Distribution of education in the two groups was not different.

Comparison of HDRS scores before administration of fluoxetine and sertraline was significantly higher in the depressed group than in the control group. Comparison of BDNF levels before administration of fluoxetine and sertraline was lower in the depressed group than in the control group. In depressed patients who were given fluoxetine therapy and whose changes were better with increased BDNF levels in the fluoxetine group than in the sertraline group. Comparison of HDRS scores after 6 weeks of therapy, there was a decrease in both the fluoxetine and sertraline groups. In fluoxetine, the results of the HDRS score show a decrease, as does the BDNF level which is increasing. Anti-depressant drugs can increase BDNF levels by activating the $5-\mathrm{HT}$ receptor so that 
serotonin levels in the presynaptic increase and activating the serotonin receptor. Periodic monitoring of BDNF levels is needed to maintain neurons from damage due to repetitive stressors.

\section{Conclusions}

The researchers concluded that there was a decrease in HDRS scores and an increase in BDNF levels in depressed patients treated with fluoxetine and sertraline. The fluoxetine group was superior in decreasing HDRS scores in depressed patients compared to the sertraline group, and the fluoxetine group was superior in increasing the BDNF levels in depressed patients compared to the sertraline group. Further study is needed to see the effect of other antidepressant groups to the level of BDNF.

\section{Authors' Contributions}

All the authors were involved in the conception of the study STL, NAM, RN, and SS to the interpretation of the research findings and contributed to the drafting of the manuscript. All authors read and approved the final manuscript.

\section{Acknowledgments}

We want to acknowledge and thank the participants who took part in this study.

\section{References}

1. Kusumawardhani, et al. Guide to Major Depression Disorders. PDSKJI; 2013.

2. Nurmiati A. Depression of the Aspects of Neurology, Diagnosis and Management. Jakarta: University of Indonesia Medical School; 2005.

3. Ministry of Health of the Republic of Indonesia. The Role of Families Supports Community Mental Health. Indonesia: Ministry of Health of the Republic of Indonesia; 2016. p. 1-2. https://doi.org/10.18311/jeoh/2020/26134

4. Afrilia S. 9 Million People in Indonesia Experience Depression; 2017. Available from: https://www.gaya.tempo.co. [Last accessed on 2021 May 10].
5. Li G, Jing P, Liu Z, Li Z, Ma H, Tu W, et al. The beneficial effect of fluoxetine treatment against psychological stress is mediated by increasing BDNF expression in selected brain areas. Oncotarget. 2017;8(41):69527-37. https://doi.org/10.18632/ oncotarget.17891

PMid:29050222

6. Francesco M, Bonaccorso S, Ricciardi A, Scaccianoce $S$, Panaccione I, Wang L, et al. Change in BDNF serum level in patients with major depression disorder (MDD) after a 6 month treatment with sertraline, escitalopram, or venlafaxine. J Psychiatr Res. 2009;43(3):247-54. https://doi.org/10.1016/j. jpsychires.2008.03.014

PMid: 18511076

7. Ayse DB, Yazici K, Aslan E, Delialioğlu N, Taşdelen B, Acar ST, et al. Effects of fluoxetine and venlafaxine on serum brain derived neurotrophic factor levels in depressed patients. 2009;33(2):281-5. https://doi.org/10.1016/j. pnpbp.2008.11.016

PMid:19110026

8. Scabia G, Barone I, Mainardi M, Ceccarini G, Scali M, Buzzigoli E, et al. The Antidepressant Fluoxetine Acts On Energy Balance and Leptin sensitivity Via BDNF. Sci Rep. 2018;8:1781. https://doi.org/10.1038/s41598-018-19886-x

9. Sylvia DE, et al. Psychiatric Textbook. $2^{\text {nd }}$ ed. Indonesia: Medical Faculty, University of Indonesia; 2013.

10. Stahl SM. Stahl's Essential Psychopharmacology. $4^{\text {th }}$ ed. New York: Cambridge University Press; 2013.

11. Stahl SM. Antipsychotic Agents in Stahl's Essential Psychopharmacology. $3^{\text {rd }}$ ed. New York: Cambridge University Press; 2008. p. 336-41.

12. Kazuko S. Brain-Derived Neurotrophic Factor For Depression Therapeutic; 2014. p. 1-10. Available from: http://www. oustinpublishgroup.org. [Last accessed on 2021 May 10].

13. Raffaela $M$, Calabrese $F$, Bedogni $F$, Tongiorgi $E$, Fumagalli $F$, Racagni $\mathrm{G}$, et al. Chronic treatment with fluoxetine up-regulates cellular BDNF mRNA experiment in rat Dopaminergic Region. Int J Neuropsychopharmacol. 2006;9(3):307-17. https://doi. org/10.1017/s1461145705005766 PMid:16035958

14. Halaris A, Sharma A, Meresh E, Pandey G, Kang R, Hage $B$, et al. BDNF serum: A potential biomarker for major depressive disorder and antidepressant response prediction. J Depress Anxiety. 2015;4:1-8. https://doi. org/10.4172/2167-1044.1000179

15. Phillips C. Brain-derived neurotrophic factor, depression, and physicalactivity; making theneuroplasticconnection. Neural Plast. 2017;2017:7260130. https://doi.org/10.1155/2017/7260130 PMid:28928987

16. Bun-Hee L, Kim YK. The poles of BDNF in the pathophysiology of major depression Psychiatry Investig. 2010;7(4):231-5. PMid:21253405

17. Klein AB, Williamson R, Santini MA, Clemmensen C, Ettrup $A$ Rios $\mathrm{M}$, et al. Blood BDNF concentration reflect brain-tissue. BDNF level. Int J Neuropsychopharmacol. 2011;14(3):347-53. https://doi.org/10.1017/s1461145710000738 PMid:20604989

18. Björkholm C, Monteggia LM. BDNF-a Key Transducer of antidepressant effects. Neuropharmacology. 2016;102:72-9. https://doi.org/10.1016/j.neuropharm.2015.10.034 PMid:26519901

19. Owen M, Wolf J, Shelly W, Rosser R, Burke HM, Lerner GK, et al. Serum BDNF level before treatment predict SSRI response in depression. Prog Neuropsychopharmacol Biol Psychiatry. 2011;35(7):1623-30. https://doi.org/10.1016/j. 
pnpbp.2011.06.013

PMid:21749907

20. SidneyH, Gorwood P. Successful Management of major Depressive Disorder. $2^{\text {nd }}$ ed. France; 2014.

21. Arumugam V, John VS, Augustine N, Jacob T, Joy SM, Sen S, et al. The impact of antidepressant treatment on brain-derived neurotrophic factor level: An evidence-based approach through systemic review and meta-analysis. Indian J Pharmacol. 2017;49(3):236-42. https://doi.org/10.4103/ijp.ijp_700_16

PMid:29033483 